\title{
Telegenetics: a systematic review of telemedicine in genetics services
}

\author{
Jennifer S. Hilgart, BSc, MSc${ }^{1}$, Julie A. Hayward, BSc' ${ }^{1}$, Bernadette Coles, $\mathrm{MSc}^{2}$ and \\ Rachel Iredale, MA, Phd $^{3}$
}

Purpose: Telemedicine is being increasingly used in many areas of health care, particularly to reduce the barriers that rural populations face in accessing health-care services. Telemedicine may also be effectively utilized in clinical genetics services-an application that has been termed "telegenetics."

Methods: A systematic review of the literature was conducted to identify studies of genetic consultations carried out through videoconferencing so as to determine whether conclusions can be drawn about the value of telegenetics. A total of 14 articles reporting data from 12 separate studies met the inclusion criteria.

Results: In a majority of these studies, patients received their telegenetics consultation at a local clinic or outreach center, from where they communicated via a synchronous video link with a genetics practitioner. All the studies reported high levels of patient satisfaction with telegenetics, and patients were generally more receptive to telegenetics than the genetics practitioners were. The studies had limitations of small sample sizes and lack of statistical analyses.

Conclusions: This review suggests that telegenetics may be a useful tool for providing routine counseling and has the potential to evaluate pediatric patients with suspected genetic conditions. Prospective, fully powered studies of telegenetics that explore the accuracy of diagnoses and patient outcomes are needed to allow informed decisions to be made about the appropriate use of telemedicine in genetics service delivery.

Genet Med 2012:14(9):765-776

Key Words: clinical genetics; genetic counseling; systematic review; telegenetics; telemedicine
Telemedicine is the use of electronic and communication technologies for medical diagnostic, monitoring, and therapeutic purposes when distance and/or time separate the participants. ${ }^{1}$ The term telemedicine often refers to a real-time interaction between a patient and a health professional through videoconferencing rather than face to face. By enabling contact with health service providers in distant locations, telemedicine can benefit rural populations by reducing geographical barriers to accessing specialist health services. ${ }^{2-5}$ Other benefits of telemedicine include improved efficiency and reduced costs of health services. The large number of articles and reviews published in recent years about telemedicine demonstrates the increasing use of, and research into, telemedicine services.

In a review of reviews, Ekeland et al. ${ }^{6}$ identified 80 systematic reviews published between 2005 and 2009 on the impact and cost of telemedicine in various areas of health care, including psychiatry, dermatology, and diabetes. Ekeland et al. concluded that the results of these reviews were diverse; some suggested that telemedicine has positive therapeutic effects and increases efficiency within health services, ${ }^{7,8}$ while other reviews concluded that the evidence for the benefits of telemedicine was inconsistent and limited. ${ }^{9,10}$ The data relating to the cost-effectiveness of telemedicine are also mixed; a recent review found that synchronous video communication was cost effective for home care and access to on-call hospital specialists but not for the local delivery of services between hospitals and primary care. ${ }^{11}$ Despite the lack of consensus within the literature regarding the effectiveness of telemedicine in improving the quality of, or access to health care, its use remains widespread. ${ }^{12}$

Telemedicine may be particularly effective in medical specialties in which verbal interactions are a key part of the assessment process, such as in psychiatry and neurology. Research in these areas demonstrates that care via telemedicine produces outcomes comparable to those of face-to-face consultations. ${ }^{13-17}$ Genetics services are often based on counseling and may therefore also be a specialized field of health care in which telemedicine can be effectively utilized as an alternative to standard face-to-face interactions between health professionals and patients.

Referrals to genetics services are generally suggested if a patient is suspected of being at risk of, or affected by, a genetic disorder. For example, genetics specialists can diagnose a patient with symptoms of a disease or carry out a disease risk assessment or a reproductive risk assessment relating to a possible carrier status or pregnancy. This often involves several visits to a genetics clinic to undergo genetic counseling and physical evaluation, receive genetic test results, and attend follow-up appointments. In Europe and the United States, access to genetics services is often available only in urban centers, ${ }^{18,19}$ 
which means that remote populations may not have access to the information, treatment, and psychosocial support that genetics services can offer. The potential for using telemedicine in clinical genetics service delivery has been highlighted by a number of authors, ${ }^{19-21}$ who have suggested that it may enable more equitable access to genetics services for people who must otherwise travel long distances to specialist centers. Indeed, an increasing number of studies are exploring alternative models of service provision, including genetic counseling over the telephone, ${ }^{22,23}$ and genetics consultations via real-time videoconferencing, ${ }^{24}$ so as to improve equality of access, improve cost efficiency, and help to meet the increasing demand for these services.

Our systematic review aims to synthesize the evidence available to date about genetic consultations carried out via videoconferencing, a process that has been termed "telegenetics". ${ }^{44,25}$ Our aim was to determine whether conclusions can be drawn about the value of telegenetics, and to provide recommendations for further research and its adoption in clinical practice.

\section{METHODS}

\section{Search strategy}

The literature search focused on English-language articles pertaining to the use of telemedicine in clinical genetics services (see Table 1 for search key words). The following electronic databases were searched: MEDLINE, EMBASE, PsychINFO, CINAHL, British Nursing Index, Cochrane Library, and the Web of Science. Each database search was conducted during the week of 28 November 2011 to identify articles published from January 1996 up to November 2011. The Science Citation Index was searched to find articles that cited the studies included in the database search results. The reference lists of all relevant articles and other reviews were used to identify additional studies.

\section{Eligibility criteria}

Studies were included in this review if they were published in a peer-reviewed journal in English and were aimed at evaluating the effectiveness, cost, or feasibility of synchronous telemedicine consultations in any area of clinical genetics. Studies that did not include genetics consultations were excluded. Randomized controlled trials and nonrandomized pilot studies were considered for inclusion. Both quantitative and qualitative studies were eligible, but reviews, letters with no primary data, case studies, and unpublished studies were excluded.

The outcomes of interest were clinical efficacy, knowledge, perceived risk, affective outcomes (e.g., distress, anxiety, and depression), behavioral outcomes (e.g., surveillance/surgery uptake and genetic testing uptake), satisfaction, and cost of the service. All titles and abstracts were screened in accordance with the inclusion criteria. Full-text articles were obtained whenever more information was required to make a decision about inclusion. Two reviewers independently reviewed the full-text studies to determine whether they met the inclusion criteria. Any disagreement about a particular study was resolved through discussion and/or referred to a third person.

\section{Information extraction}

Two review authors independently extracted data from each of the studies included in the review. The data extracted included study design, method and setting; participant characteristics; and relevant outcomes. Any differences in opinion were resolved through discussion. Reviewers were not blinded as to authorship, journal, or institution.

\section{Quality assessment}

A formal quality assessment was not performed because a majority of the studies were nonrandomized studies with small sample sizes, whereas all well validated quality assessment tools are intended for use in the context of randomized controlled trials.

\section{Study characteristics}

\section{RESULTS}

A total of 14 articles reporting data from 12 separate studies met all the study selection criteria. Details of the studies included, published between the years 2000 and 2011, are provided in Table 2 . The studies were conducted in the United States, Canada, the UK, and Australia. All the studies utilized real-time videoconferencing via multiple high-speed ISDN lines (between 128 and 384 kbps) to provide clinical genetics services to remote or outreach areas. ISDN lines are considered to be effective in transmitting high-resolution images and are reasonably fast and secure. In a majority of the studies, patients received their telegenetics consultations at a local clinic or outreach center. Exceptions to this were Meropol et al., ${ }^{26}$ a study in which the participants were counseled in their own homes, and Gattas et al. ${ }^{27}$ in which both the telegenetics group and the face-to-face group had their consultations in the same hospital in Brisbane, Australia, so as to remove the advantages of travel time and cost.

The studies included in our review related to the use of telegenetics in counseling for hereditary cancer, ${ }^{26,28-33}$ prenatal counseling, ${ }^{34}$ pediatric services, ${ }^{35,36}$ and services for a range of genetic disorders. ${ }^{24,27,37}$ Of the 125 patients who were evaluated and/or counseled via telemedicine in the study by Lea et al., ${ }^{37}$ $64 \%$ received pediatric and/or neurological genetics consultations and the rest received consultations for cancer or reproductive genetics counseling. Pediatric telegenetics services often included physical examinations, whereas consultations for cancer generally only involved a review of the patients' family history, the provision of information, and psychosocial support. The type of genetics practitioner involved in the telemedicine consultations varied between studies. In several studies, patients had a genetics counselor or nurse present with them at the outreach clinic while they communicated via a synchronous video link with a geneticist or a genetics counselor. ${ }^{24,27,30-33,38}$ In a study by Stalker et al., ${ }^{36}$ a genetics counselor obtained information on patients' family and medical history via teleconferencing, and a local pediatrician conducted a physical examination. The 
Table 1 Review search strategy

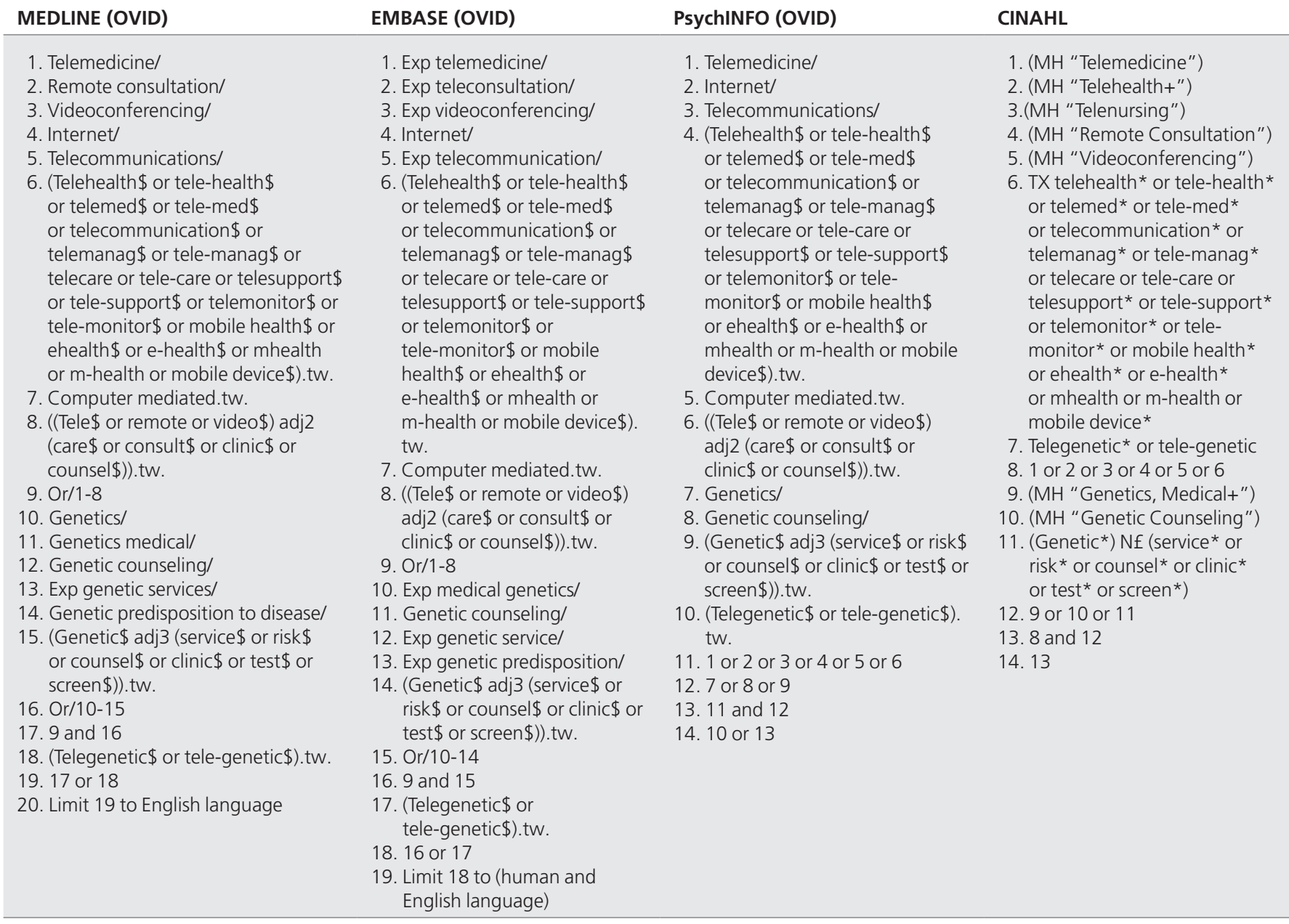

patients then returned to the clinic for a face-to-face consultation with the clinical geneticist. Three of the studies explored genetics counseling via teleconference with a genetics counselor only; these comprised one study involving prenatal counseling ${ }^{34}$ and two studies involving hereditary cancer counseling. ${ }^{26,29}$ Coelho et al., ${ }^{28}$ described a study of genetics counseling for cancer via teleconference with a genetics consultant.

Only one of the studies in our review involved randomization of participants to receive either a telegenetics consultation or a face-to-face consultation, with both groups attending the same hospital for their appointments. ${ }^{27}$ Patients in the telegenetics group communicated via a video link with the consultant, who was located only 10 meters away. The majority of the studies used a retrospective or cross-sectional design, with evaluations and assessments occurring after the telegenetics consultation. Three studies were prospective studies with assessments both before and after telegenetics counseling. . $4,28,33^{2}$ Two studies involved conducting qualitative interviews, one with patients ${ }^{32}$ and the other with genetics professionals, ${ }^{30,31}$ to explore their experiences of telegenetics. The other studies involved the gathering of data through surveys and/or telephone interviews, and many did not involve any statistical analyses on outcomes measures because of small sample sizes. Only one study indicated that validated measurement tools with acceptable reliability had been used to assess outcomes. ${ }^{33}$ That study was also the only one to mention that power calculations were conducted to ensure that any significant between-group differences were detected. Eight of the studies did not have a comparison group, and reported data solely on those who received genetics services via a telemedicine..$^{24,26,29-32,35-38}$ All the studies measured patient satisfaction with the telegenetics consultation; in some cases, the health professionals' satisfaction was also assessed. The methods of measuring satisfaction varied between studies, with many researchers developing their own scales of satisfaction, whereas others based their scales on previous research. Three studies reported on affective outcomes such as cancer-specific distress, general anxiety, and depression. ${ }^{24,28,33}$ There were no reports on clinical or behavioral outcomes such as uptake of genetics testing or cancer screening after telegenetics counseling for hereditary cancer. However, Lea et al. ${ }^{37}$ Hopper et al., ${ }^{35}$ and Stalker et al. ${ }^{36}$ reported on the clinical efficacy of diagnosing pediatric genetic disorders through the use of telegenetics. 


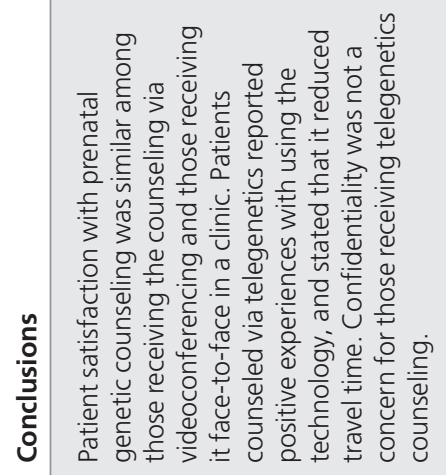

高

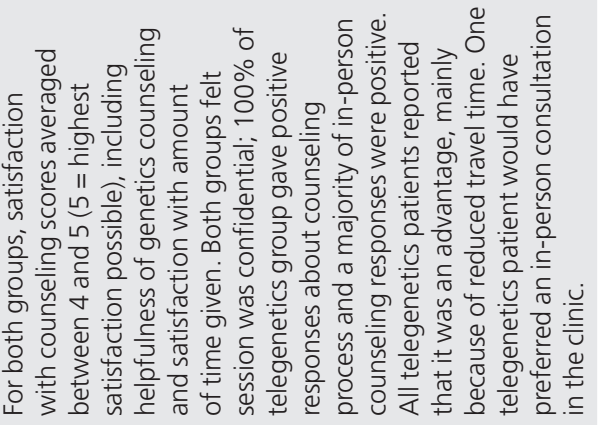

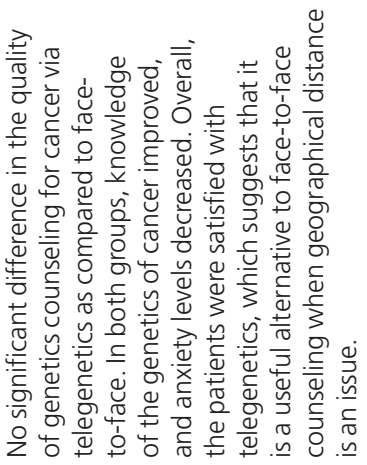

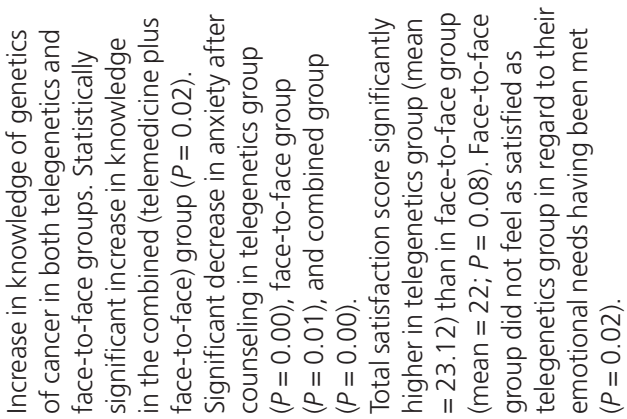

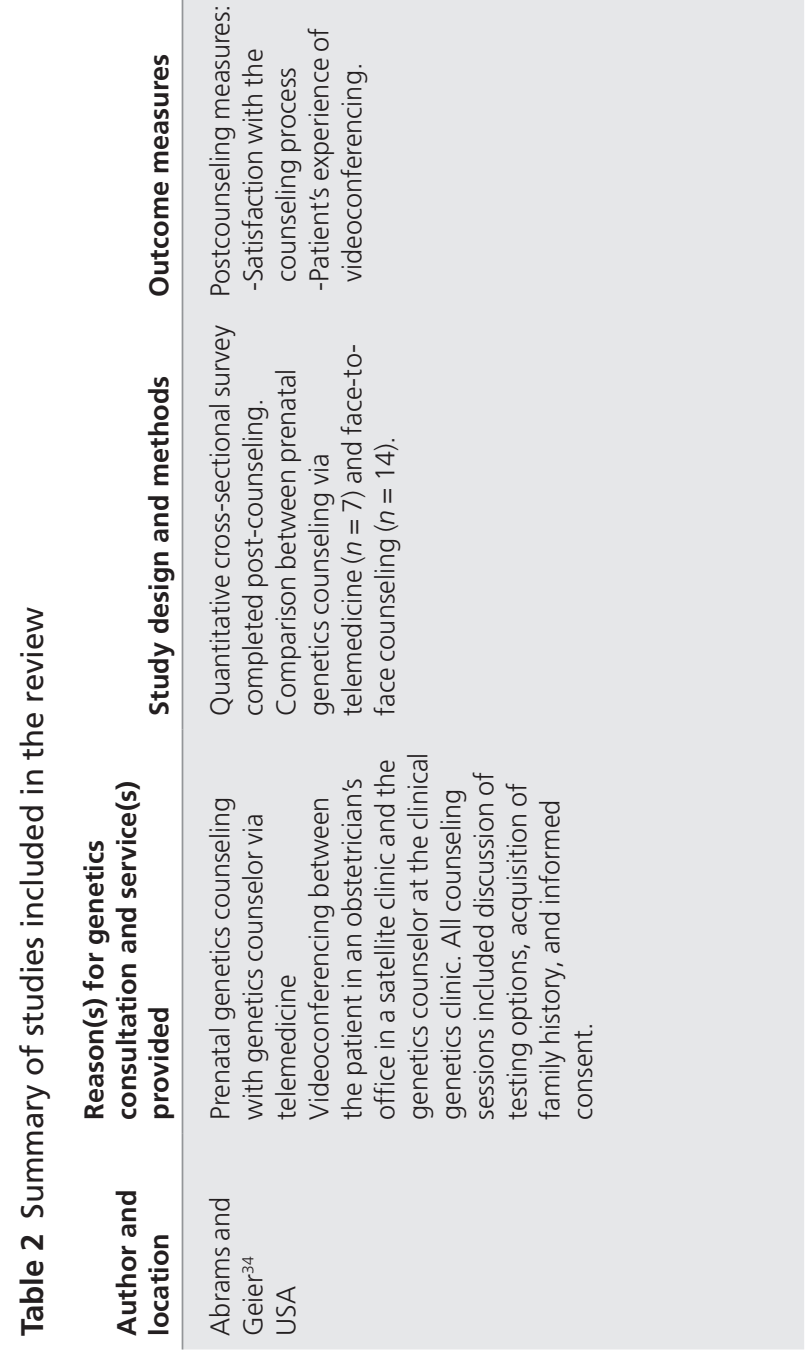
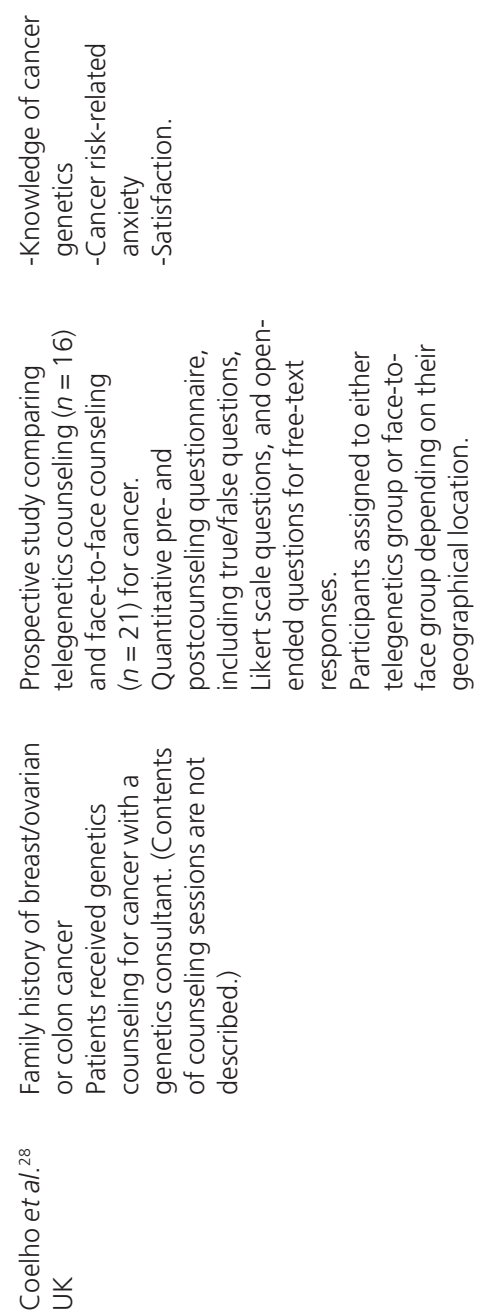


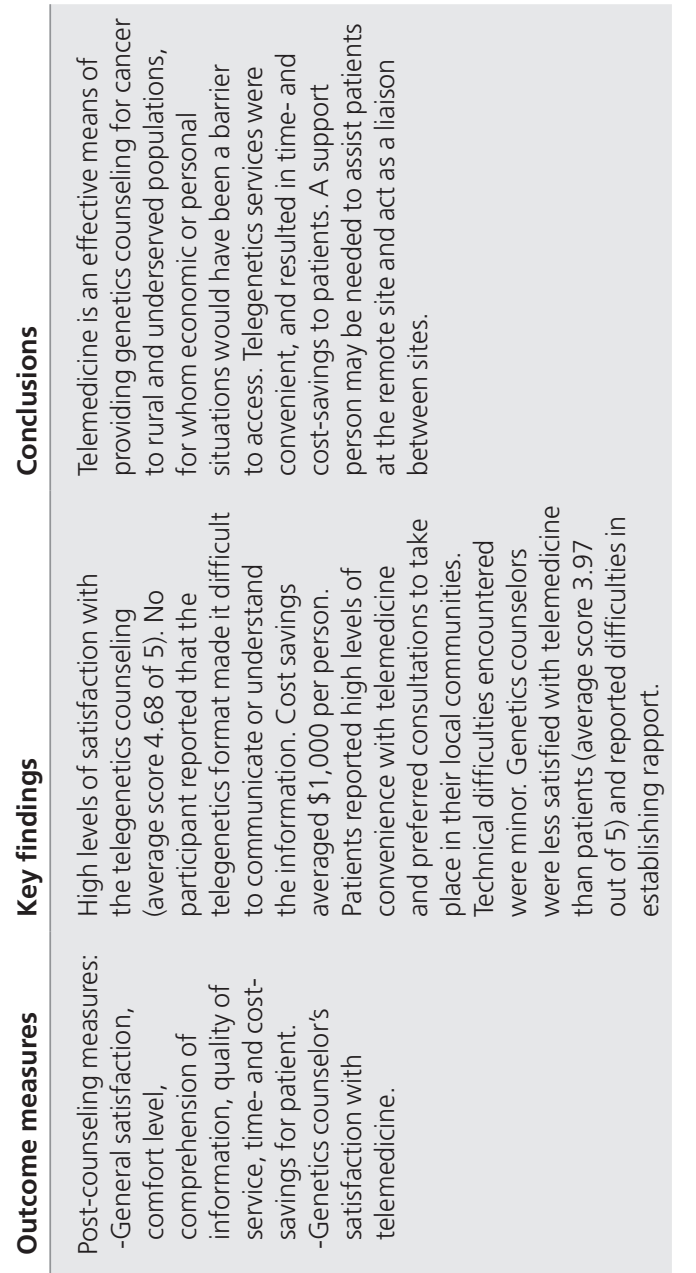

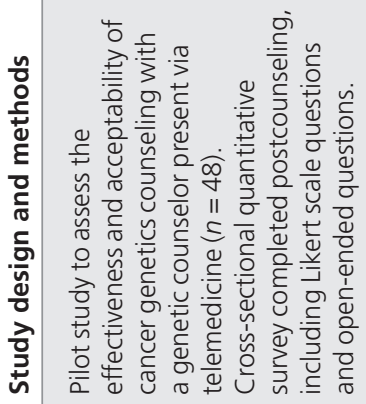

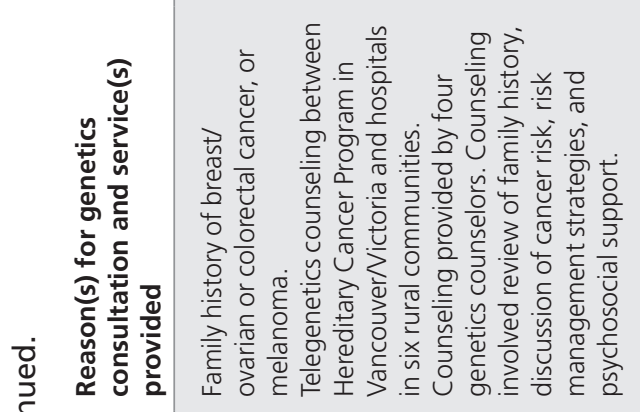

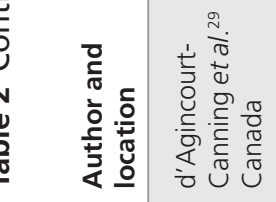

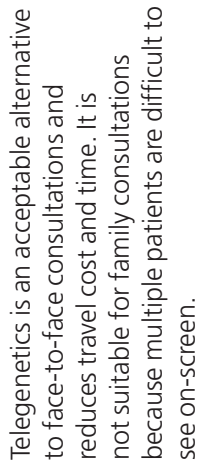

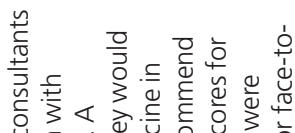

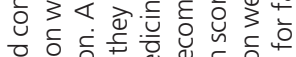

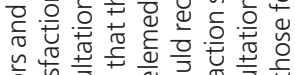

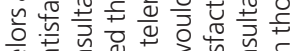

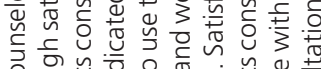

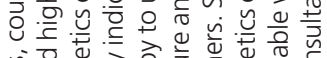

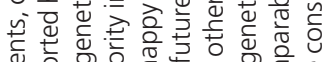

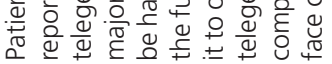
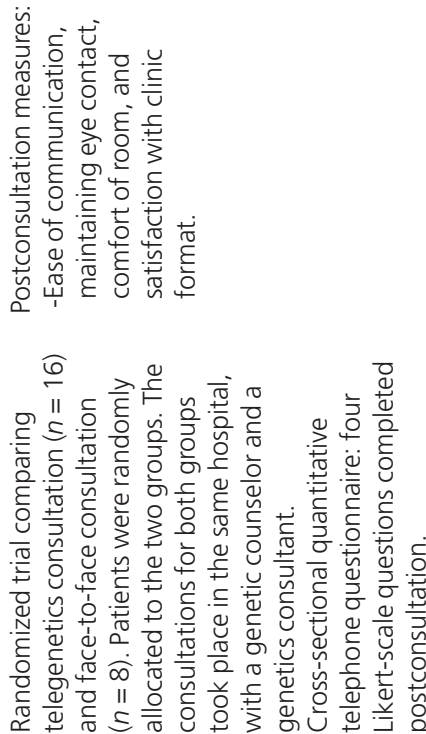

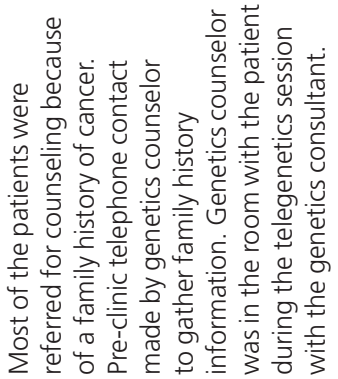

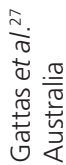

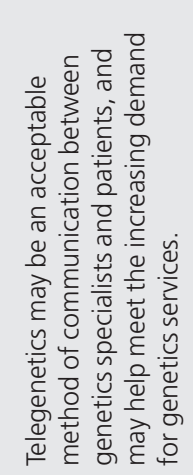

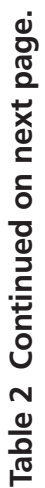

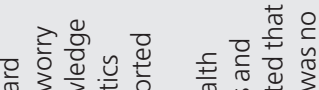

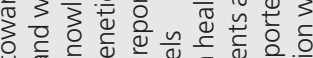

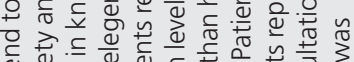

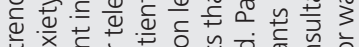

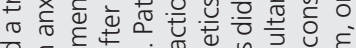
o $\subseteq$ ह

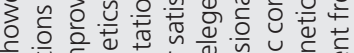

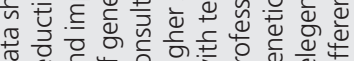

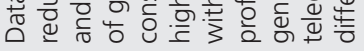
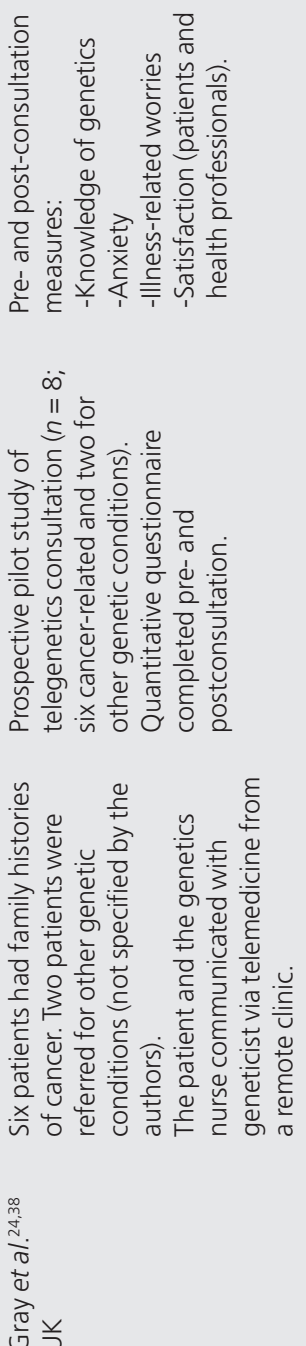


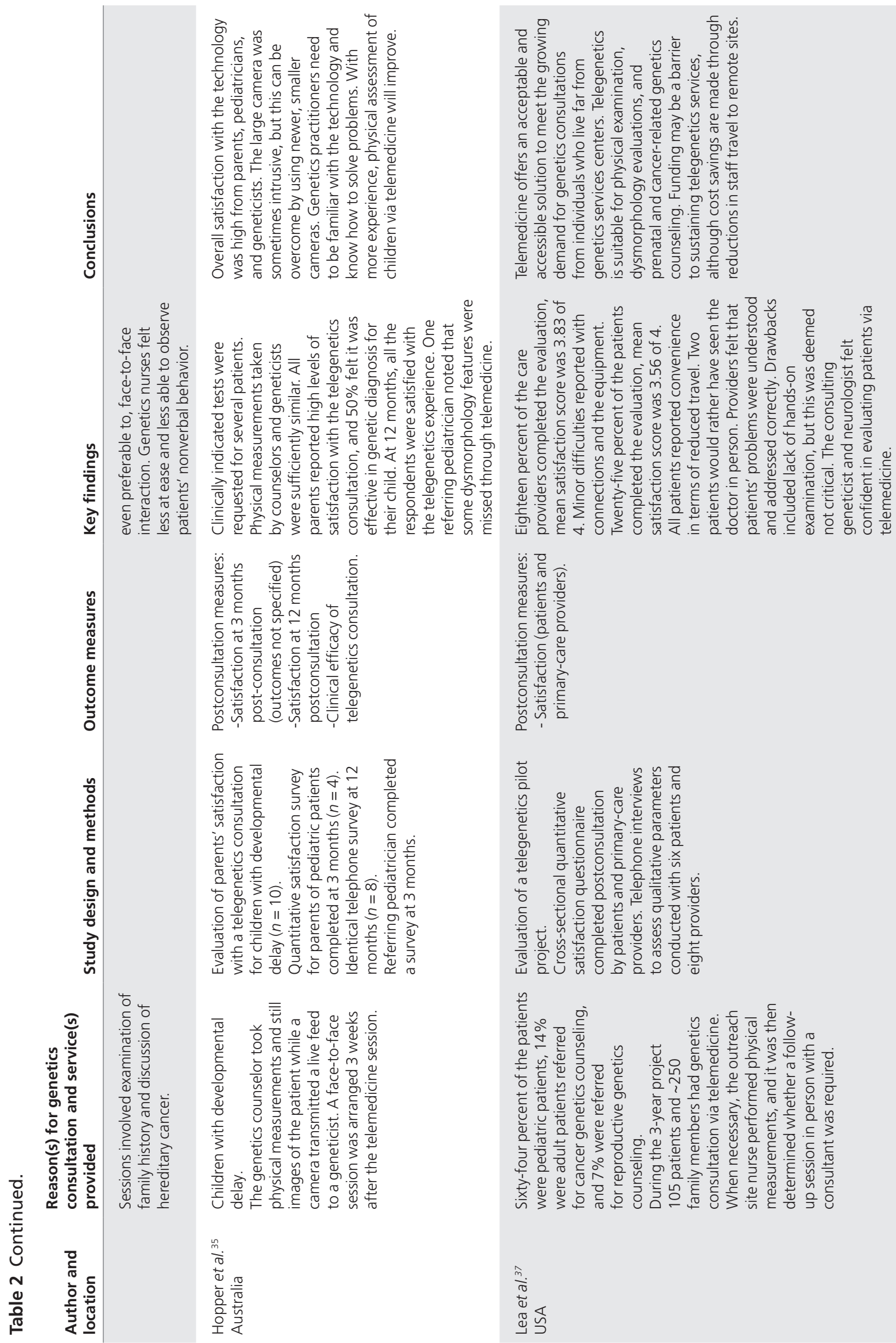




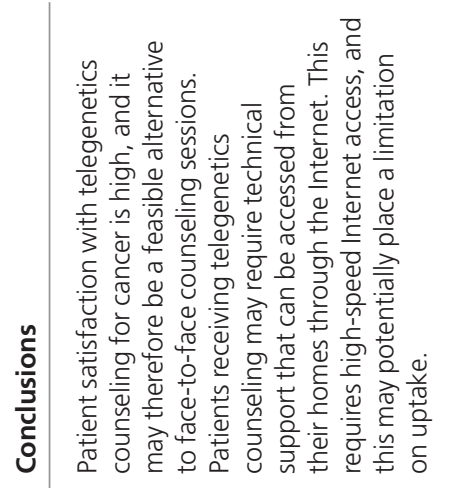

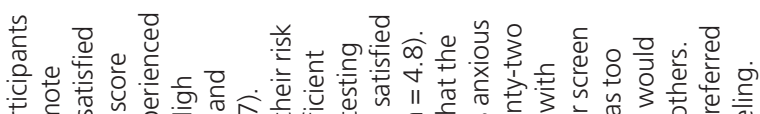

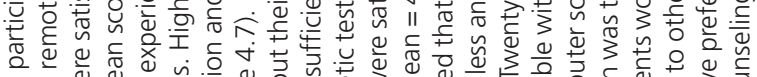

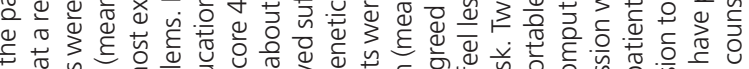

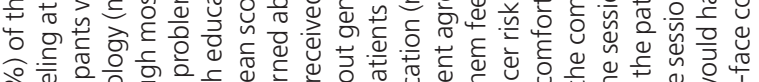
⿹ 口

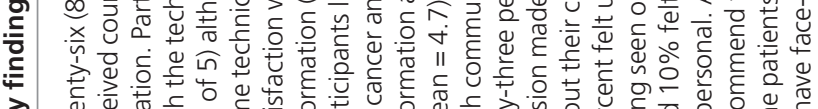
离
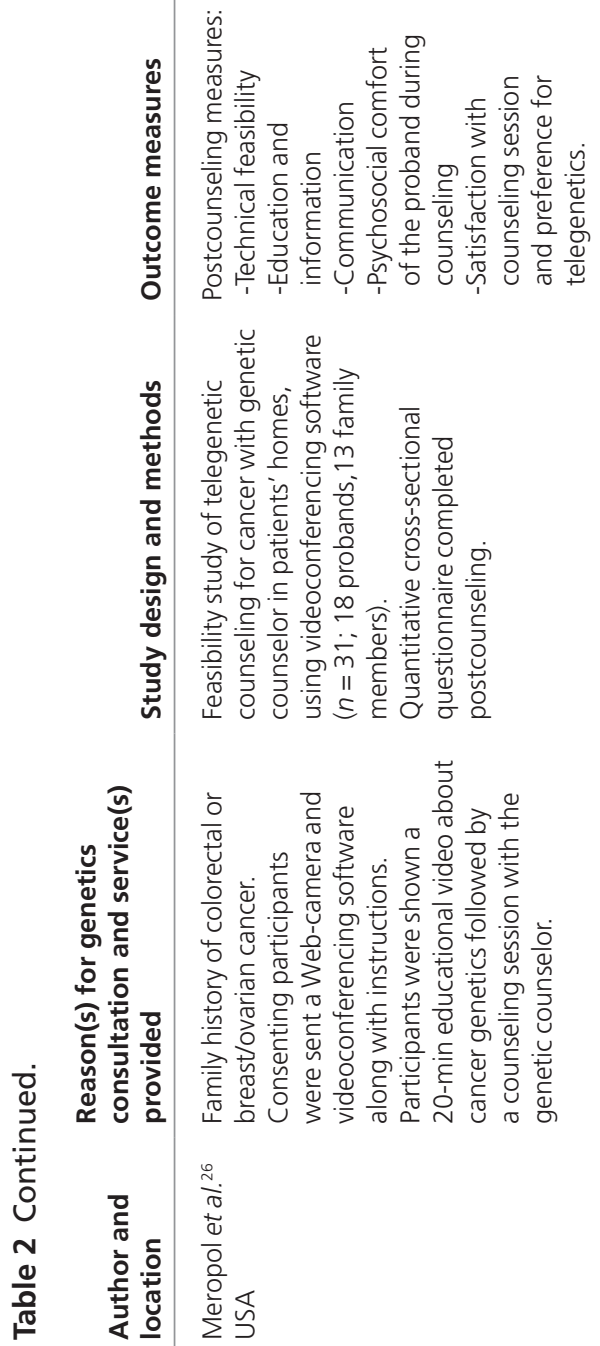

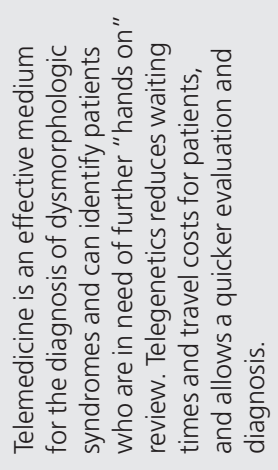

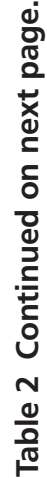
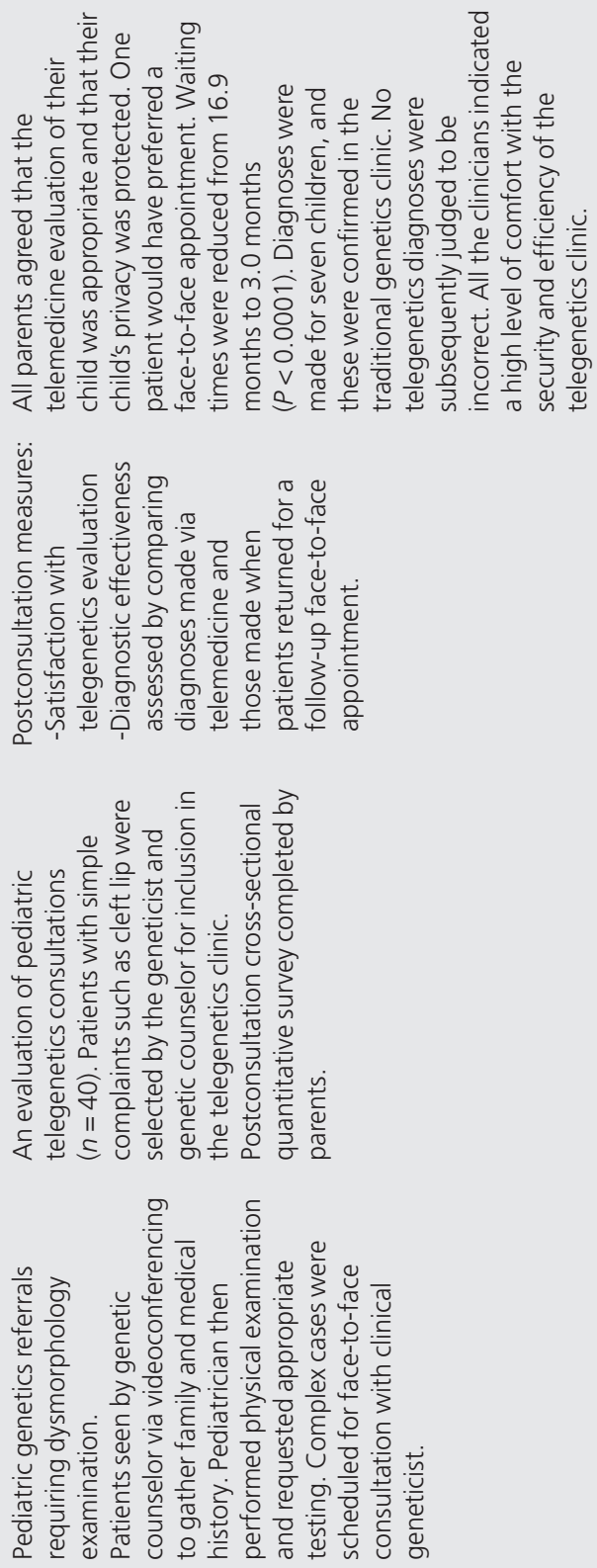

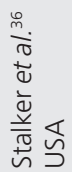



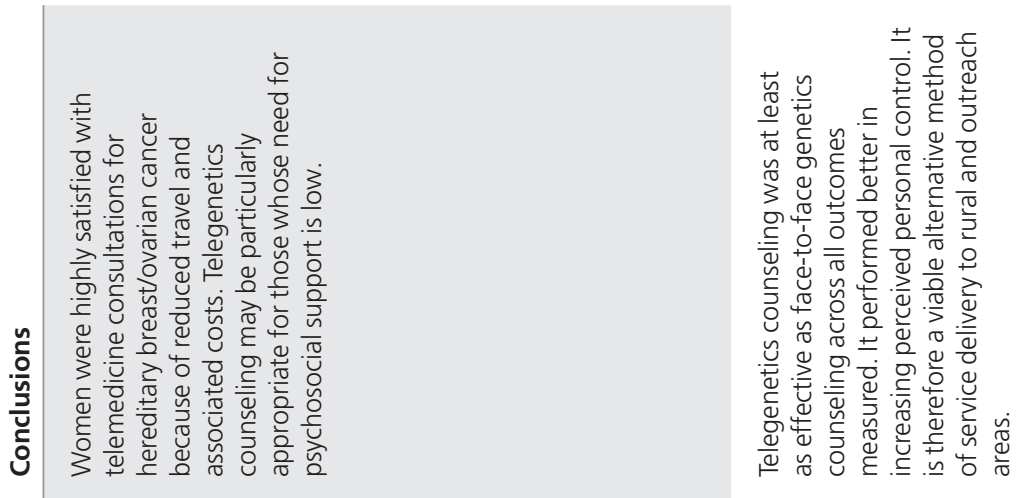

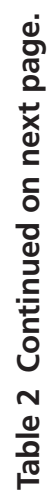

产

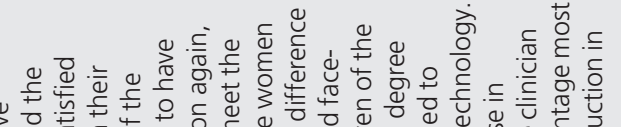

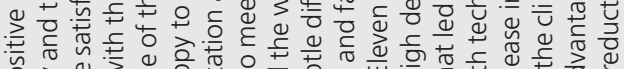

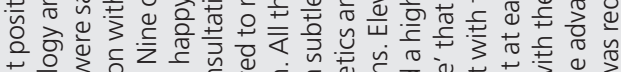

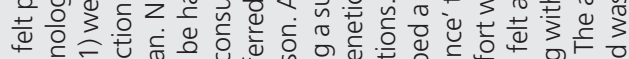

и

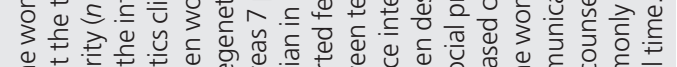

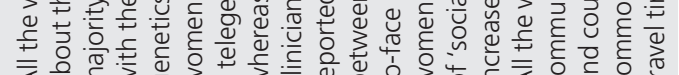

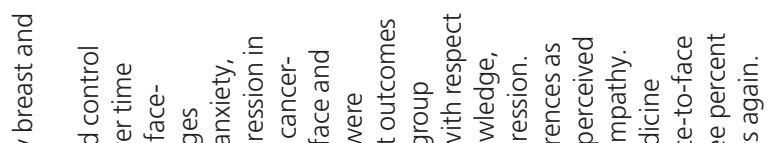

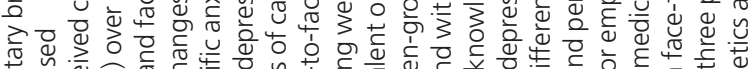

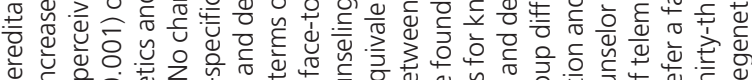

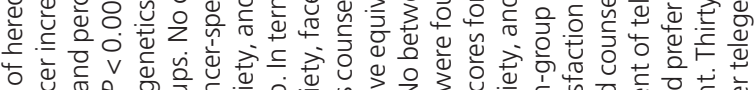

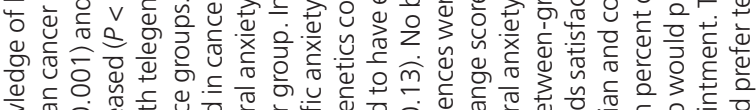

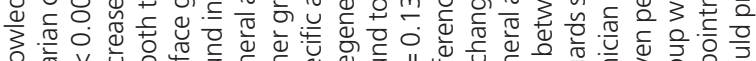

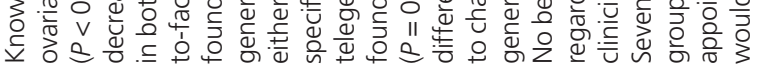
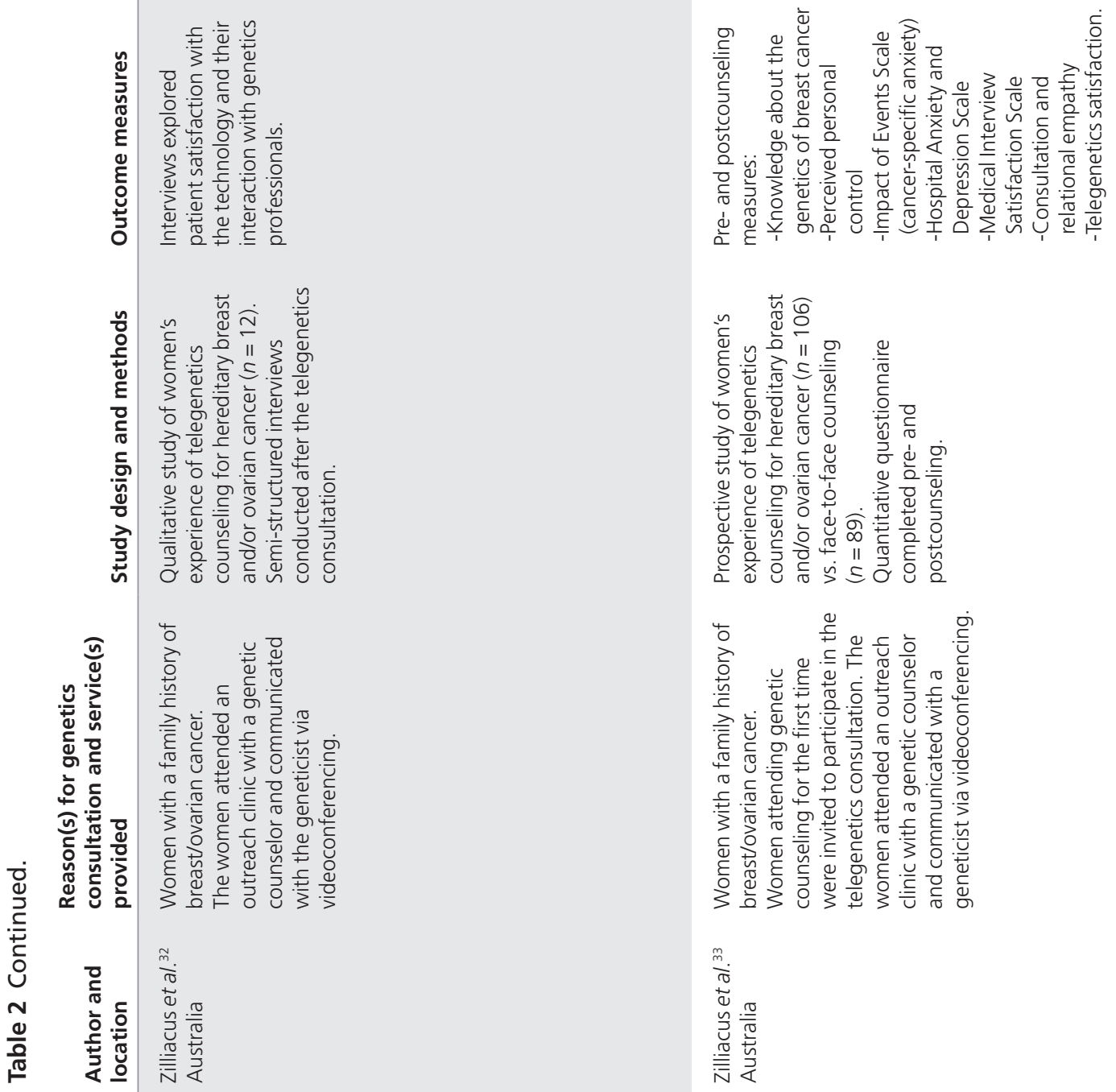
Overall, the studies included in this review are heterogeneous and vary in quality. Most of the studies had limitations of small sample sizes that precluded the use of statistical analyses. The limitations also included retrospective study designs and the lack of comparison groups, and the data were therefore subject to bias.

\section{Satisfaction with telegenetics services}

All the studies reported high levels of patient satisfaction with the telegenetics services, as ascertained through questionnaires or interviews. Patients were generally satisfied with the technology used, the education and information provided, and the opportunity to communicate with genetics professionals without having to travel long distances. Those involved in the care of pediatric patients felt that telemedicine was an effective medium through which to evaluate genetic conditions in their children. ${ }^{35-37}$ Where a comparison group was utilized, the satisfaction levels of those receiving telegenetics services were generally no different from those in the group receiving face-toface counseling. Indeed, in one study, the satisfaction level in the telegenetics group was higher than in the control group. ${ }^{28}$ The studies reported several benefits of telegenetics services: convenience, reduced travel time and associated costs, and reduced waiting times to see a genetics specialist. ${ }^{36}$ Patients tended to be more satisfied than the genetics practitioners with telegenetics counseling. ${ }^{24,29,37,38}$ Patients viewed the technical difficulties involved as being less problematic, and were more satisfied with the rapport established with the genetics professional.

In a study by Gray et al. ${ }^{24,38}$ nursing staff expressed dissatisfaction with telegenetics because they felt excluded from the geneticist-patient interaction and less able to observe the nonverbal behavior of patients. Similarly, in a study by Zilliacus et al. ${ }^{30,31}$ genetics practitioners reported that there were disadvantages to telegenetics counseling for patients with hereditary breast/ovarian cancer, including inhibition of rapport between the geneticist and the patient, and difficulty for the practitioner in detecting nonverbal cues from patients. Patients may have been more satisfied with telegenetics consultations because of the savings in travel time and costs; a majority of the patients preferred to receive genetic counseling services in a local setting. In many studies, patients who received telegenetics counseling were asked whether they would have preferred to have a face-to-face appointment. Women receiving genetic counseling for cancer would be happy to have another telegenetics consultation, but a majority $(n=7)$ wanted to meet the geneticist in person. ${ }^{32}$ A study by Meropol et al. ${ }^{26}$ reported that all the participants would recommend telegenetics counseling to other patients, although nine of them (29\%) agreed that they would have preferred a face-to-face session. In Abrams and Grier's ${ }^{34}$ study of prenatal telegenetics counseling, only one participant would have preferred an in-person counseling session instead.

\section{Affective outcomes}

The three studies that reported on affective outcomes of telegenetics consultations used prospective study designs. All three 
studies reported that patients' knowledge of genetics increased after the telegenetics consultations. Gray et al. ${ }^{24}$ reported that general anxiety and cancer-specific anxiety decreased over time in those who received telegenetics counseling. Coelho et al. ${ }^{28}$ found significant decreases in anxiety levels after cancer genetics counseling in the telegenetics group $(P=0.00)$, the face-to-face group $(P=0.01)$, and the combined (telemedicine and face-to-face) group $(P=0.00)$. Zilliacus et al.$^{33}$ reported no change in cancer-specific anxiety, general anxiety, or depression in either the telegenetics group or the face-to-face group in a cohort of women receiving genetics counseling for hereditary breast/ovarian cancer. In studies that compared telegenetics groups with those receiving face-to-face consultations, there were no significant between-group differences in any of the affective outcome variables. ${ }^{28,33}$

\section{Clinical efficacy}

Three of the studies explored the effectiveness of telegenetics in diagnosing genetics-related conditions in pediatric patients. ${ }^{35-37}$ In a study by Stalker et al. ${ }^{36}$ a genetics counselor ascertained the family history and a pediatrician performed a physical examination to screen for potential genetic syndromes. The first eight patients evaluated via telemedicine were also assessed in person by a clinical geneticist. Diagnoses were made for seven of the children via telegenetics, and these were confirmed in the faceto-face genetics clinic. No new diagnoses were made in the faceto-face sessions that had not been identified in the telegenetics sessions, and none of the diagnoses made through telegenetics were deemed inaccurate. The authors concluded that telegenetics was successful in identifying individuals in need of further hands-on dysmorphologic review and in providing quicker confirmation of the absence of a particular genetic syndrome. Lea et al. ${ }^{37}$ also reported that telegenetics was effective as a dysmorphology screening tool, because patients could be referred for an in-person evaluation if a dysmorphologic genetic syndrome was suspected. However, in a study by Hopper et al., ${ }^{35}$ one referring pediatrician was of the opinion that several physical characteristics could potentially be missed in a telegenetics consultation. In that study, the genetics counselor took physical measurements and still images of the patients while a camera transmitted a live feed to the clinical geneticist. Face-to-face sessions were arranged 3 weeks after the telegenetics sessions. The measurements taken in person by the genetics counselors and those ascertained by the clinical geneticist from the live feeds of images varied slightly but did not affect either the assessments or the diagnoses. The authors of the study concluded that, with an increase in shared telegenetics experience between the genetics counselor and the geneticist, dysmorphologic assessment of children via telegenetics consultations will improve.

\section{Costs of telegenetics}

None of the studies included in our review formally measured the costs of telegenetics services. Most of the studies reported that patients made savings in terms of travel time and costs by receiving telegenetics services rather than face-to-face genetics services. In one study, telegenetics was estimated to confer an average saving of $\$ 1,000$ per person. ${ }^{29}$ The same authors also concluded that videoconferencing is less expensive than outreach programs. They estimated that, in Canada, it would cost $\$ 700$ for a genetics counselor to travel to a specific site to provide outreach care for 12 patients at a 2 -day clinic. In contrast, the costs associated with the same number of appointments through videoconferencing are negligible, provided the necessary equipment is already available and in place. ${ }^{29}$

\section{DISCUSSION}

Our review shows that patients are generally highly satisfied with the use of telemedicine in genetics service delivery, including genetics counseling and diagnoses of pediatric genetic conditions. The studies included in the review also show that the affective outcomes for patients receiving telegenetics consultations are comparable to those for patients receiving face-to-face consultations. This suggests that telegenetics is acceptable to patients and does not appear to have any detrimental effects on patient anxiety. Neither the type of health professional involved in the telemedicine consultation nor the presence of a genetics nurse/counselor at the outreach site appeared to affect patient satisfaction. Comparable results were reported from the various studies conducted in different countries. The opinions of genetics practitioners regarding telegenetics services were not always as positive, and many expressed concerns about the difficulty in establishing a rapport with patients via telemedicine and difficulties associated with the use of the technology. Many of the studies concluded that, with appropriate training and growing experience with telegenetics services, genetics practitioners will become more accepting of the technology. In a study by Lea et al., ${ }^{37}$ practitioners rated telegenetics services higher after they had personally provided these services, suggesting that familiarity and confidence with using the technology will reduce practitioners' resistance to adopting telegenetics services. Although most patients appreciated the fact that telegenetics counseling had the benefits of reduced waiting times and/or shorter travel distances, there is a need to be cautious when interpreting data from patient surveys regarding telegenetics consultations. These patients have received a service they ordinarily would not have, and they may therefore feel obligated to endorse it. ${ }^{39}$ This is especially relevant in studies wherein patients were allowed to choose whether to receive telegenetics services or face-to-face services (e.g., the studies by d'Agincourt-Canning et al., ${ }^{29}$ and Meropol et al. ${ }^{26}$ Indeed, previous research has found that patients who were able to choose how they received their BRCA1/2 genetic test result-whether by telephone or in person-reported higher levels of satisfaction than patients who did not have a choice. ${ }^{22}$ Studies with random assignment to telegenetics services or faceto-face services may help to reduce this response bias.

It could also be argued that the selection of new referrals for telegenetics consultations may introduce some bias into research results (e.g., the study by Stalker et al. ${ }^{36}$ ). However, many studies have stated that telegenetics is not intended to replace existing services but may be a useful adjunct to 
traditional service delivery for dysmorphologic evaluation and/ or genetics consultations. In the study by d'Agincourt-Canning et al. ${ }^{29}$ some participants reported that they would not have followed through with their referral to the Hereditary Cancer Program had they been unable to have an appointment in their local area. Telemedicine may therefore extend access to remote populations and reduce waiting times to consult with genetics specialists, and thereby increase the capacity to provide genetics services to those who require them and not just to those fortunate enough to live close to genetics centers. ${ }^{21,39}$

The cost-utility assessment of providing telegenetics services, including costs of equipment, transmission lines, training, and personnel has not been formally carried out, and is a vital area for further research. High-quality evidence on the efficacy and cost-effectiveness of telegenetics will encourage the expansion of these services within the National Health Service in the UK. In the United States, reimbursement of telegenetics costs needs to be sufficient in order to provide sustainable telegenetics clinics. This involves ensuring that telegenetics has the support of health insurers, not only the private insurance companies but also Medicare and Medicaid, which are the federal health insurers for the elderly and poor. To date, Medicaid does not universally reimburse for telemedicine consultations in all 50 states, nor do private payers. Under Medicare programs there are also wide variations in service coverage, payment policies, and other stipulations ${ }^{40}$ and these are considered to be barriers to the long-term sustainability and expansion of telemedicine services. ${ }^{41-43}$ Whitten and Buis ${ }^{41}$ suggest that, in order to promote more consistent reimbursement for telemedicine services, it is essential that both consumer demand and provider acceptance for these services increase. They also suggest that demonstrating the clinical effectiveness of telemedicine will encourage reimbursement for these services in the long term. Our review has demonstrated that patients are generally satisfied with telegenetics, but that further research is needed to explore beyond self-reported measures of patient satisfaction and fully investigate patient outcomes so as to determine whether quality of care is affected by the type of consultation received. ${ }^{44,45}$

\section{Conclusions}

Although the studies relating to the use of telegenetics are few in number to date and generally small in sample size, they suggest that telegenetics may be appropriate for providing additional genetics services to reduce inequalities in access to genetics specialists. Telemedicine technology is being utilized in many medical specialties; teledermatology, telepsychiatry, and teleradiology are already being used, and the delivery of genetics services through telegenetics could be a feasible approach. The studies included in this review suggest that telegenetics may not only be a useful tool to provide routine counseling and follow-up appointments, but that it also has the potential to evaluate and diagnose pediatric patients with suspected genetic conditions. Prospective, fully powered, and well designed studies of telegenetics services, assessing the accuracy of diagnoses, the impact of receiving a diagnosis this way, and patient outcomes are needed to make informed decisions about the appropriate use of telemedicine in genetics service delivery.

\section{ACKNOWLEDGMENT}

This review was funded by Tenovus, the cancer charity in Wales.

\section{DISCLOSURE}

The authors declare no conflict of interest.

\section{REFERENCES}

1. Institute of Medicine (U.S.). Committee on evaluating clinical applications of telemedicine. Telemedicine: A Guide to Assessing Telecommunications in Health Care. National Academy Press: Washington, DC, 1996.

2. Malasanos TH, Burlingame JB, Youngblade L, Patel BD, Muir AB. Improved access to subspecialist diabetes care by telemedicine: cost savings and care measures in the first two years of the FITE diabetes project. J Telemed Telecare 2005;11(suppl 1):74-76

3. Raza T, Joshi M, Schapira RM, Agha Z. Pulmonary telemedicine-a model to access the subspecialist services in underserved rural areas. Int J Med Inform 2009;78:53-59.

4. Sevean P, Dampier S, Spadoni M, Strickland S, Pilatzke S. Patients and families experiences with video telehealth in rural/remote communities in Northern Canada. J Clin Nurs 2009;18:2573-2579.

5. Saeed SA, Diamond J, Bloch RM. Use of telepsychiatry to improve care for people with mental illness in rural North Carolina. N C Med J 2011;72:219-222.

6. Ekeland AG, Bowes A, Flottorp S. Effectiveness of telemedicine: a systematic review of reviews. Int J Med Inform 2010;79:736-771.

7. Clark RA, Inglis SC, MCAlister FA, Cleland JG, Stewart S. Telemonitoring or structured telephone support programmes for patients with chronic heart failure: systematic review and meta-analysis. BMJ 2007;334:942.

8. Spek V, Cuijpers P, Nyklícek I, Riper H, Keyzer J, Pop V. Internet-based cognitive behaviour therapy for symptoms of depression and anxiety: a meta-analysis. Psychol Med 2007;37:319-328.

9. Verhoeven F, van Gemert-Pijnen L, Dijkstra K, Nijland N, Seydel E, Steehouder $\mathrm{M}$. The contribution of teleconsultation and videoconferencing to diabetes care: a systematic literature review. J Med Internet Res 2007;9:e37.

10. Postel MG, de Haan HA, De Jong CA. E-therapy for mental health problems: a systematic review. Telemed J E Health 2008;14:707-714.

11. Wade VA, Karnon J, Elshaug AG, Hiller JE. A systematic review of economic analyses of telehealth services using real time video communication. BMC Health Serv Res 2010;10:233.

12. Hersh WR, Hickam DH, Severance SM, Dana TL, Krages KP, Helfand M. Diagnosis, access and outcomes: update of a systematic review of telemedicine services. J Telemed Telecare 2006;12(suppl 2):3-31.

13. Chua R, Craig J, Esmonde T, Wootton R, Patterson V. Telemedicine for new neurological outpatients: putting a randomized controlled trial in the context of everyday practice. J Telemed Telecare 2002;8:270-273.

14. Meyer BC, Raman R, Hemmen T, et al. Efficacy of site-independent telemedicine in the STRokE DOC trial: a randomised, blinded, prospective study. Lancet Neuro/ 2008;7:787-795.

15. Mitchell JE, Crosby RD, Wonderlich SA, et al. A randomized trial comparing the efficacy of cognitive-behavioral therapy for bulimia nervosa delivered via telemedicine versus face-to-face. Behav Res Ther 2008;46:581-592.

16. Ruskin PE, Silver-Aylaian M, Kling MA, et al. Treatment outcomes in depression: comparison of remote treatment through telepsychiatry to in-person treatment. Am J Psychiatry 2004;161:1471-1476.

17. O'Reilly R, Bishop J, Maddox K, Hutchinson L, Fisman M, Takhar J. Is telepsychiatry equivalent to face-to-face psychiatry? Results from a randomized controlled equivalence trial. Psychiatr Serv 2007;58:836-843.

18. Washington State Department of Health, Genetic Services Policy Project Final Report, 2008. https://depts.washington.edu/genpol/docs/AppD.pdf. Accessed 10 October 2011.

19. Godard B, Kääriäinen H, Kristoffersson U, Tranebjaerg L, Coviello D, Aymé S. Provision of genetic services in Europe: current practices and issues. Eur $J$ Hum Genet 2003;11(suppl 2):S13-S48.

20. Lea DH. A new world view of genetics service models. Online J Issues Nurs 2000;5:5.

21. Hawkins AK, Hayden MR. A grand challenge: providing benefits of clinical genetics to those in need. Genet Med 2011;13:197-200. 
22. Baumanis L, Evans JP, Callanan N, Susswein LR. Telephoned BRCA1/2 genetic test results: prevalence, practice, and patient satisfaction. $J$ Genet Couns 2009;18:447-463.

23. Jenkins J, Calzone KA, Dimond E, et al. Randomized comparison of phone versus in-person BRCA1/2 predisposition genetic test result disclosure counseling. Genet Med 2007;9:487-495.

24. Gray J, Brain K, Iredale R, Alderman J, France E, Hughes H. A pilot study of telegenetics. J Telemed Telecare 2000;6:245-247.

25. Mitchell JA, Demiris G. Telegenetics: the next phase in the provision of genetics services? Genet Med 2005;7:1-2.

26. Meropol NJ, Daly MB, Vig HS, et al. Delivery of Internet-based cancer genetic counselling services to patients' homes: a feasibility study. J Telemed Telecare 2011;17:36-40

27. Gattas MR, MacMillan JC, Meinecke I, Loane M, Wootton R. Telemedicine and clinical genetics: establishing a successful service. J Telemed Telecare 2001;7(suppl 2):68-70.

28. Coelho JJ, Arnold A, Nayler J, Tischkowitz M, MacKay J. An assessment of the efficacy of cancer genetic counselling using real-time videoconferencing technology (telemedicine) compared to face-to-face consultations. Eur J Cancer 2005;41:2257-2261.

29. d'Agincourt-Canning L, McGillivray B, Panabaker K, et al. Evaluation of genetic counseling for hereditary cancer by videoconference in British Columbia. B C Med J 2008;50:554-559.

30. Zilliacus E, Meiser B, Lobb E, Dudding TE, Barlow-Stewart K, Tucker $K$. The virtual consultation: practitioners' experiences of genetic counseling by videoconferencing in Australia. Telemed J E Health 2010;16:350-357.

31. Zilliacus E, Meiser B, Lobb E, Barlow-Stewart K, Tucker K. A balancing act-telehealth cancer genetics and practitioners' experiences of a triadic consultation. J Genet Couns 2009;18:598-605.

32. Zilliacus EM, Meiser B, Lobb EA, Kirk J, Warwick L, Tucker K. Women's experience of telehealth cancer genetic counseling. J Genet Couns 2010;19:463-472.

33. Zilliacus EM, Meiser B, Lobb EA, et al. Are videoconferenced consultations as effective as face-to-face consultations for hereditary breast and ovarian cancer genetic counseling? Genet Med 2011;13:933-941.
34. Abrams DJ, Geier MR. A comparison of patient satisfaction with telehealth and on-site consultations: a pilot study for prenatal genetic counseling. J Genet Couns 2006;15:199-205.

35. Hopper B, Buckman M, Edwards M. Evaluation of satisfaction of parents with the use of videoconferencing for a pediatric genetic consultation. Twin Res Hum Genet 2011;14:343-346.

36. Stalker HJ, Wilson R, McCune H, Gonzalez J, Moffett M, Zori RT. Telegenetic medicine: improved access to services in an underserved area. J Telemed Telecare 2006;12:182-185.

37. Lea DH, Johnson JL, Ellingwood S, Allan W, Patel A, Smith R. Telegenetics in Maine: Successful clinical and educational service delivery model developed from a 3-year pilot project. Genet Med 2005;7:21-27.

38. Iredale R, Gray J, Murtagh G. Telegenetics: A pilot study of video-mediated genetic consultations in Wales. Int J Med Market 2002;2:130-135.

39. Elliott AM, Mhanni AA, Marles SL, et al. Trends in Telehealth versus On-site Clinical Genetics Appointments in Manitoba: A Comparative Study. J Genet Couns 2012;21:337-344

40. Brown NA. State Medicaid and private payer reimbursement for telemedicine: An overview. J Telemed Telecare 2006;12(suppl 2): S32-S39.

41. Whitten $P$, Buis $L$. Private payer reimbursement for telemedicine services in the United States. Telemed J E Health 2007;13:15-23.

42. Schmeida M, McNeal R, Mossberger K. Policy determinants affect telehealth implementation. Telemed J E Health 2007;13:100-107.

43. California Telemedicine and ehealth Center. Telemedicine reimbursement: A national scan of current policies and emerging initiatives, 2009. http://www.cteconline.org/_pdf/CTEC-National-Scan. pdf. Accessed 31 October 2011.

44. Woods KF, Kutlar A, Johnson JA, et al. Sickle cell telemedicine and standard clinical encounters: a comparison of patient satisfaction. Telemed J 1999;5:349-356.

45. Moher D, Cook DJ, Eastwood S, Olkin I, Rennie D, Stroup DF. Improving the quality of reports of meta-analyses of randomised controlled trials: the QUOROM statement. Quality of Reporting of Meta-analyses. Lancet 1999;354:1896-1900. 